Artículo científico

Volumen 30(2):447-458. Mayo-agosto, 2019 e-ISSN 2215-3608, doi:10.15517/am.v30i1.34145 https://revistas.ucr.ac.cr/index.php/agromeso/index

\title{
Evaluación de cámaras de recuento sobre parámetros espermáticos de verracos analizados con un sistema CASA-Mot ${ }^{1}$
}

\section{Assessment of counting chambers on boar sperm parameters analyzed by a CASA-Mot system}

\author{
Anthony Valverde ${ }^{2,3}$, Mónica Madrigal-Valverde ${ }^{2,4}$
}

1 Recepción: 3 de julio, 2018. Aceptación: 17 de octubre, 2018. Este trabajo formó parte del proyecto de investigación "CASA-Mot Technology” desarrollado por el primer autor en el Departamento de Biología Celular y Antropología Física de la Universidad de Valencia, España.

2 Instituto Tecnológico de Costa Rica, Escuela de Agronomía, Centro de Investigación y Desarrollo en Agricultura Sostenible del Trópico Húmedo (CIDASTH), Campus San Carlos. Apdo. Postal 223-21001 Alajuela, Costa Rica. anvalverde@tec.ac.cr (autor para correspondencia, https://orcid.org/0000-0002-3191-6965); mmadrigal@itcr.ac.cr

3 Universidad de Valencia, Facultad de Ciencias Biológicas, Campus Burjassot. C/ Dr. Moliner 50, 46100 Valencia, España.

4 Universidade Federal da Bahia, Escola de Veterinaria e Zootecnia. CEP 40170-110, Bahía, Brasil.

\section{Resumen}

Introducción. La comprensión de la variabilidad en los valores de la cinética de los espermatozoides, por medio de diferentes profundidades de cámaras, demuestra la importancia de crear un estándar para los métodos de control de calidad en la industria de la inseminación artificial (IA). Objetivo. El objetivo del presente trabajo fue evaluar los parámetros de cinética de los espermatozoides con base en diferentes profundidades de cámara de visualización mediante un sistema comercial de análisis de esperma asistidos por computadora, CASA-Mot. Materiales y Métodos. Se utilizaron veinte dosis seminales de diez verracos pietrain. El periodo experimental fue de febrero a julio del año 2017. Se utilizó el sistema integrado de análisis de semen (ISAS®V1) con una frecuencia de captura de $50 \mathrm{~Hz}$. Se utilizaron cámaras de recuento ISAS ${ }^{\circledR} 4 \mathrm{C} 16$ e ISAS ${ }^{\circledR} \mathrm{D} 4 \mathrm{C} 20$ con una altura de 16 y $20 \mu \mathrm{m}$, respectivamente y precalentadas a $37{ }^{\circ} \mathrm{C}$. Resultados. Se encontraron valores superiores $(\mathrm{p}<0,05)$ para todos los parámetros cinéticos cuando la altura de la cámara de recuento fue de $20 \mu \mathrm{m}$. El efecto de zona dentro de la cámara de recuento se mantuvo constante entre las dos alturas, y las variaciones observadas en los parámetros cinéticos se debieron a un efecto aleatorio del verraco. Al analizar el efecto de zona dentro de la cámara de recuento, los primeros tres campos de análisis mostraron mayor velocidad curvilínea y rectilínea $(\mathrm{p}<0,05)$ que los siguientes campos, lo que se atribuye a la presencia de movimiento pasivo (drifting). Conclusión. La mayor amplitud y capacidad de volumen dentro de la cámara de recuento $(20 \mu \mathrm{m}$ versus $16 \mu \mathrm{m})$, podría favorecer el desplazamiento sin restricciones de las células, lo que explicaría el incremento en los valores cinéticos conforme aumentó la altura de la cámara. Deben continuarse los estudios sobre las condiciones técnicas del análisis seminal para estandarizar los métodos de valoración con los sistemas CASA.

Palabras clave: semen, verraco, espermatozoide, reproducción. 


\begin{abstract}
Introduction. Understanding the variability in sperm kinetic values through different chambers depths, shows the importance to create a standard for quality control methods in the artificial insemination (AI) industry. Objective. The work aimed was to evaluate the spermatozoa kinetic parameters based on different depths of the visualization chamber by means of a commercial system of computer-assisted sperm analysis, CASA-Mot. Materials and Methods. Twenty seminal doses of ten pietrain boars were used. The experimental period was from February to July 2017. The Integrated Semen Analyses System (ISAS ${ }^{\circ} v 1$ ) with $50 \mathrm{~Hz}$ capture frequency was used. ISAS ${ }^{\circledR}$ D 416 and ISAS ${ }^{\oplus} 4 \mathrm{C} 20$ counting chambers with a height of 16 and $20 \mu \mathrm{m}$ respectively and pre-heated to $37^{\circ} \mathrm{C}$ were employed. Results. Higher values $(p<0.05)$ were found for all kinetic parameters when the height of the counting chamber was $20 \mu \mathrm{m}$. The zone effects within the counting chamber were constant between the two heights, and the variations observed in the kinetic parameters were due to a random effect of the boar. When analyzing the zone effect within the counting chamber, the first three fields of analysis showed higher curvilinear and rectilinear velocity $(\mathrm{p}<0.05)$ than the following fields, which is attributed to the presence of passive movement (drifting). Conclusion. The greater amplitude and volume capacity within the counting chamber $(20 \mu \mathrm{m}$ versus $16 \mu \mathrm{m})$, could promote the unrestricted movement of the cells, which would explain the increase in the kinetic values as the chamber height increased. Studies on the technical conditions of seminal analysis should be continued in order to standardize valuation methods with CASA systems.
\end{abstract}

Keywords: semen, boar, spermatozoa, reproduction.

\title{
Introducción
}

Desde que se iniciaron los estudios sobre calidad seminal, se ha puesto de manifiesto que en la evaluación del semen la movilidad y cinética espermática son los dos factores más importantes. La utilización de sistemas computarizados de análisis de semen (Computer Assisted Semen Analysis, CASA) implica un avance en la estandarización de dichas valoraciones, pero requieren de la validación de protocolos adecuados que optimicen sus resultados (World Health Organization, 2010).

Aunque se ha demostrado que los sistemas CASA son más precisos que los métodos tradicionales (Krause, 1995; Broekhuijse et al., 2011), pueden producirse variaciones en los resultados que induzcan al error, incluso cuando se utiliza la determinación computarizada de la movilidad espermática (Amann y Waberski, 2014; Simonik et al., 2015). Los resultados pueden verse influenciados por una serie de fuentes de variación, como la configuración del software, el número de imágenes capturadas, el número de campos analizados, la dilución de la muestra, la temperatura de la muestra y el tiempo transcurrido desde la eyaculación hasta el examen (Broekhuijse et al., 2011). Otros factores como la estandarización de los equipos utilizados, pueden influenciar los resultados (Verstegen et al., 2002), junto a la aptitud del técnico (Ehlers et al., 2011), el tipo de software (Tejerina et al., 2008) y el tipo de cámara utilizada para el análisis (Kraemer et al., 1998; Tomlinson et al., 2001; Lenz et al., 2011; Hoogewijs et al., 2012; Gloria et al., 2013).

La determinación precisa de las variables cinemáticas de los espermatozoides resulta fundamental para la correcta valoración de la calidad seminal. Las determinaciones realizadas con el uso de cámaras de recuento, pueden estimarse con la utilización de sistemas CASA (Bompart et al., 2018). Sin embargo, los sistemas CASAMot no siguen un patrón estándar común y los informes de movilidad generados con un sistema comercial pueden no ser directamente comparables con los obtenidos usando otros sistemas (Gill et al., 1988; Jasko et al., 1990; Olds-Clarke et al., 1990; Hoogewijs et al., 2012). Los efectos de los ajustes en la configuración (Smith y England, 
2001; Rijsselaere et al., 2003), como el número de imágenes por segundo (frame rate), el número de imágenes analizadas y la cámara de recuento, son algunos de los factores que afectan los resultados finales. La literatura que informa del análisis de semen, describe una amplia variedad de metodologías tanto en términos de entornos CASAMot (Loomis y Graham, 2008; Waite et al., 2008; Ortega-Ferrusola et al., 2009), así como en el uso de diferentes cámaras de recuento en combinación con sistemas CASA (Ortega-Ferrusola et al., 2009). Los sistemas CASA-Mot requieren la utilización de cámaras de recuento específicas y es necesario comprender las propiedades asociadas con cada tipo de cámara (Lannou et al., 1992; Massányi et al., 2008; Hoogewijs et al., 2012). En todo caso, la literatura científica no ha desarrollado oportunamente la valoración de los diferentes tipos y alturas de cámara disponibles en el mercado. Además, se debe considerar que independientemente del método que se utilice para determinar los parámetros espermáticos, siempre se parte de la base de que se toma una submuestra de la muestra total, por lo que se realiza una estimación del parámetro real en la población (Douglas-Hamilton et al., 2005).

En el mercado se encuentran disponibles diversos tipos de cámaras para utilizar con los sistemas CASA. También, se existe variabilidad en el diseño, forma y tamaño de las cámaras disponibles. Estas diferencias, pueden condicionar el eventual análisis seminal, afectando la dinámica de los espermatozoides de una manera diferente, lo que conduce a valores de salida divergentes. Con el propósito de que los resultados del análisis de semen tengan mayor precisión y exactitud, es fundamental la elección del tipo de cámara que ofrezca el mayor grado de confianza. El objetivo del presente trabajo fue evaluar los parámetros de cinética de los espermatozoides con base en diferentes profundidades de cámara de visualización mediante un sistema comercial de análisis de esperma asistidos por computadora, CASA-Mot.

\section{Materiales y métodos}

\section{Descripción y localización del experimento}

Todos los análisis de movilidad y cinética espermática se desarrollaron en el laboratorio de andrología de Proiser R+D, ubicada en el parque científico de la Universidad de Valencia, Campus de Burjassot, Valencia, España. El periodo experimental fue de febrero a julio del año 2017. Las muestras de semen se mantuvieron en condiciones controladas de temperatura y almacenamiento en el laboratorio, con el propósito de no introducir fuentes de variación en la movilidad seminal.

\section{Animales}

Se utilizaron veinte dosis seminales comerciales, obtenidas de diez verracos donadores de semen de la raza pietrain, con edades entre 2 y 3 años. Estos animales fueron alojados en edificios con clima controlado en la granja comercial de verracos Semen Cardona, S.L. (Cardona, Barcelona, España). Los verracos se alimentaban con una dieta comercial para verracos reproductores ajustada $\left(2,3 \mathrm{~kg} \cdot \mathrm{d}^{-1}\right)$ y provistos de agua ad libitum. Las muestras fueron transportadas al laboratorio en las mismas condiciones de refrigeración $\left(17^{\circ} \mathrm{C}\right)$ utilizadas para la distribución comercial. Al llegar al laboratorio las muestras se agitaron y posteriormente se tomó una alícuota de $1 \mathrm{ml}$, se colocaron en tubos Eppendorf y se mantuvieron a $37{ }^{\circ} \mathrm{C}$ durante $30 \mathrm{~min}$ antes de su análisis espermático.

\section{Evaluación de las variables espermáticas}

Para el análisis de parámetros cinéticos, se utilizaron cámaras de recuento desechables, precalentadas a $37^{\circ} \mathrm{C}$, basadas en el principio de difusión capilar, ISAS ${ }^{\circledR}$ D4C16 e ISAS ${ }^{\circledR}$ D 4 C20 (Proiser R + D, S.L., Paterna, España). 
Las alturas correspondientes de ambas cámaras fueron 16 y $20 \mu \mathrm{m}$, respectivamente. Después de homogenizar las muestras, se distribuyó un volumen de semen de $3 \mu \mathrm{l}$ a lo largo de la cámara de conteo por capilaridad. Los análisis se realizaron con el sistema comercial de análisis de esperma asistidos por computadora, el sistema CASA-Mot ISAS ${ }^{\circledR} v 1$ (Proiser R + D). La cámara de video fue Proiser HS640m (Proiser R + D), con una frecuencia de captura de 50 imágenes por segundo (frame per second, fps) y una resolución final de 640 x 478 píxeles. La cámara se conectó a un microscopio UB203 (UOP / Proiser R + D) con un ocular de 1X, un objetivo de contraste de fase negativo $10 \mathrm{X}(\mathrm{AN} 0,25)$ y una placa calefactora integrada mantenida a $37 \pm 0,5{ }^{\circ} \mathrm{C}$. El resto de la configuración del equipo considerada fue la que venía indicada por defecto del fabricante para el análisis de muestras de verraco. Tanto las imágenes como los datos se guardaron para su posterior reevaluación. Se aleatorizó el orden en que se hicieron los análisis, para evitar que influyera de forma constante en los resultados obtenidos. El llenado de las cámaras se realizó por capilaridad, por lo que pudo darse diferentes distribuciones celulares en función del avance capilar de la muestra. En este caso, las posibles diferencias deberían aparecer a lo largo del recorrido, analizándose las posibles modificaciones desde la posición 1 hasta la 7. La altura del espacio en que se mueven los espermatozoides condiciona la movilidad y la distribución de los mismos, por lo que se analizó el efecto de dicha característica sobre los resultados obtenidos.

\section{Análisis de parámetros cinéticos}

Cada muestra se analizó por duplicado y se tomaron como mínimo siete campos del microscopio con un total de al menos 600 células analizadas por muestra. Se utilizó una media de siete campos para los análisis estadísticos. Los parámetros CASA-Mot considerados en el estudio fueron: la velocidad rectilínea $\left(\mathrm{VSL}, \mu \mathrm{m} \cdot \mathrm{s}^{-1}\right)$, correspondiente a la línea recta desde el principio hasta el final de la trayectoria; la velocidad curvilínea (VCL, $\left.\mu \mathrm{m} \cdot \mathrm{s}^{-1}\right)$, medida sobre la trayectoria real de punto a punto seguida por el espermatozoide; la velocidad de trayectoria promedio (VAP, $\mu \mathrm{m} \cdot \mathrm{s}^{-1}$ ), calculada como una interpolación entre los puntos correspondientes a la trayectoria de la VCL; la amplitud de desplazamiento lateral de la cabeza (ALH, $\mu \mathrm{m})$, expresada como la altura máxima (o media) de la amplitud del movimiento oscilatorio de la trayectoria curvilínea; y la frecuencia de batido cruzado (BCF, $\mathrm{Hz}$ ), expresada como el número de veces que la trayectoria curvilínea cruza la lineal. La configuración del software se ajustó al análisis de espermatozoides de verraco: de 10 a $80 \mu \mathrm{m}^{2}$, para el área de la cabeza, y 11 para conectividad. Además, se estimaron tres relaciones de progresión expresadas como porcentajes, a partir de las mediciones de velocidad descritas anteriormente: linealidad de la progresión directa $(\mathrm{LIN}=\mathrm{VSL} / \mathrm{VCL} * 100)$, rectitud $(\mathrm{STR}=$ VSL / VAP * 100) e índice de oscilación (WOB = VAP / VCL *100).

\section{Diseño experimental}

En este estudio se analizaron veinte eyaculados (dos eyaculados por cada verraco) y tres réplicas por eyaculado de diez verracos raza pietrain. Todas las muestras de semen se registraron a una frecuencia de captura de $50 \mathrm{fps}$ durante $1 \mathrm{~s}$. Este experimento se realizó a tiempo de análisis cero para todas las alturas de cámara.

\section{Análisis estadístico}

Se evaluó la normalidad mediante el papel probabilístico normal y la homocedasticidad mediante la prueba de Levene. Se utilizó un ANOVA de una vía para determinar el efecto de la altura de la cámara de recuento sobre los parámetros cinéticos. En los casos pertinentes, se aplicó la prueba de Bonferroni para el análisis a posteriori de las variaciones entre alturas de cámara. Los resultados se presentaron como medias \pm error estándar de la 
media (SEM). La significancia estadística se consideró en p $\leq 0,05$. Los análisis estadísticos se realizaron usando Statgraphics Centurion XVII, 17.2.04. (32-bit) (1982-2016 por Statpoint Technologies Inc., USA).

\section{Resultados}

En cuanto a los parámetros VCL y VSL se observó que para las cámaras ISAS ${ }^{\circledR}$ D4C16 e ISAS ${ }^{\circledR}$ D4C20, los valores obtenidos en los diferentes campos de las cámaras de recuento, no hubo diferencias $(\mathrm{p}>0,05)$ en los primeros cuatro campos, mientras que en los campos quinto a sétimo sí hubo diferencia $(p<0,05)$ entre ellos. No se presentaron diferencias en la linealidad medida con la cámara de $16 \mu \mathrm{m}(\mathrm{p}>0,05)$ mientras que con la altura de 20 $\mu \mathrm{m}$, hubo diferencias $(\mathrm{p}<0,05)$ entre los campos analizados en las zonas extremas de la cámara de recuento (Cuadro 1). En promedio, la altura de cámara de $16 \mu \mathrm{m}$ mostró menos diferencias $(\mathrm{p}<0,05)$ entre los animales que con la cámara de $20 \mu \mathrm{m}$ para todos los campos dentro de la cámara de recuento. En el primer caso, solo cuatro animales fueron diferentes entre sí y con respecto del total, mientras que en el segundo caso seis animales presentaron diferencias (Figura 1). Las variaciones observadas entre las zonas de análisis dentro de la cámara de recuento por animal fueron efecto aleatorio del verraco para los parámetros cinéticos. Se observó una tendencia generalizada a presentar mayores velocidades en la zona 1 (primer campo de captura), como consecuencia de la presencia de movimiento pasivo (drifting, Figura 1).

Cuadro 1. Parámetros de velocidad y linealidad espermática (medias $\pm E E$ ) en espermatozoides de verraco raza pietrain medidos en diferentes zonas de la cámara de recuento seminal y en dos tipos de cámara. Valencia, España. 2017.

Table 1. Velocity and linearity sperm parameters (means \pm SE) on boar spermatozoa pietrain race measured at different areas of the counting chamber and two types of camera. Valencia, Spain. 2017.

\begin{tabular}{|c|c|c|c|}
\hline Campo/variable & VCL & VSL & LIN \\
\hline \multicolumn{4}{|c|}{ ISAS $^{\circledR} \mathrm{D} 4 \mathrm{C} 16$} \\
\hline 1 & $107,68 \pm 1,46^{\mathrm{a}}$ & $34,28 \pm 0,48^{\mathrm{a}}$ & $37,42 \pm 0,56^{\mathrm{a}}$ \\
\hline 2 & $103,46 \pm 1,36^{\mathrm{a}}$ & $34,60 \pm 0,44^{\mathrm{a}}$ & $39,21 \pm 0,52^{\mathrm{a}}$ \\
\hline 3 & $103,66 \pm 1,27^{\mathrm{a}}$ & $32,91 \pm 0,41^{\mathrm{a}}$ & $38,65 \pm 0,49^{a}$ \\
\hline 4 & $94,35 \pm 1,39^{\mathrm{b}}$ & $28,07 \pm 0,45^{\mathrm{b}}$ & $37,56 \pm 0,54^{\mathrm{a}}$ \\
\hline 5 & $92,73 \pm 1,63^{\mathrm{bc}}$ & $29,12 \pm 0,53^{\mathrm{b}}$ & $39,11 \pm 0,63^{\mathrm{a}}$ \\
\hline 6 & $87,40 \pm 1,81^{\mathrm{c}}$ & $28,11 \pm 0,59^{b}$ & $39,23 \pm 0,70^{\mathrm{a}}$ \\
\hline 7 & $88,97 \pm 1,77^{\text {bc }}$ & $29,18 \pm 0,58^{\mathrm{b}}$ & $38,97 \pm 0,68^{\mathrm{a}}$ \\
\hline \multicolumn{4}{|c|}{ ISAS $^{\circledR}$ D4C20 } \\
\hline 1 & $109,13 \pm 1,23^{\mathrm{b}}$ & $41,43 \pm 0,45^{\mathrm{a}}$ & $44,71 \pm 0,51^{\mathrm{ab}}$ \\
\hline 2 & $110,77 \pm 1,19^{\mathrm{ab}}$ & $40,09 \pm 0,44^{\mathrm{a}}$ & $41,98 \pm 0,50^{\mathrm{cd}}$ \\
\hline 3 & $109,77 \pm 1,22^{\mathrm{ab}}$ & $39,84 \pm 0,45^{\mathrm{ab}}$ & $42,62 \pm 0,51^{\mathrm{bc}}$ \\
\hline 4 & $114,50 \pm 1,16^{\mathrm{a}}$ & $39,86 \pm 0,43^{\mathrm{ab}}$ & $40,86 \pm 0,49^{\mathrm{cd}}$ \\
\hline 5 & $107,90 \pm 1,90^{\mathrm{b}}$ & $38,21 \pm 0,44^{\mathrm{bc}}$ & $41,63 \pm 0,50^{\mathrm{cd}}$ \\
\hline 6 & $93,04 \pm 1,11^{\mathrm{d}}$ & $37,16 \pm 0,41^{\mathrm{c}}$ & $46,27 \pm 0,46^{\mathrm{a}}$ \\
\hline 7 & $99,05 \pm 1,16^{\mathrm{c}}$ & $33,52 \pm 0,42^{\mathrm{d}}$ & $39,94 \pm 0,49^{\mathrm{d}}$ \\
\hline
\end{tabular}

VCL: velocidad curvilínea; VSL: velocidad rectilínea; LIN: índice de linealidad. a,b,c,d Dentro de columna, valores con diferente superíndice presentan diferencias significativas $(\mathrm{p}<0,05)$ / VCL: curvilinear velocity; VSL: rectilinear velocity; LIN: linearity index. a,b,c,d Within the column, values with different superscripts show significant differences $(\mathrm{p}<0.05)$. 

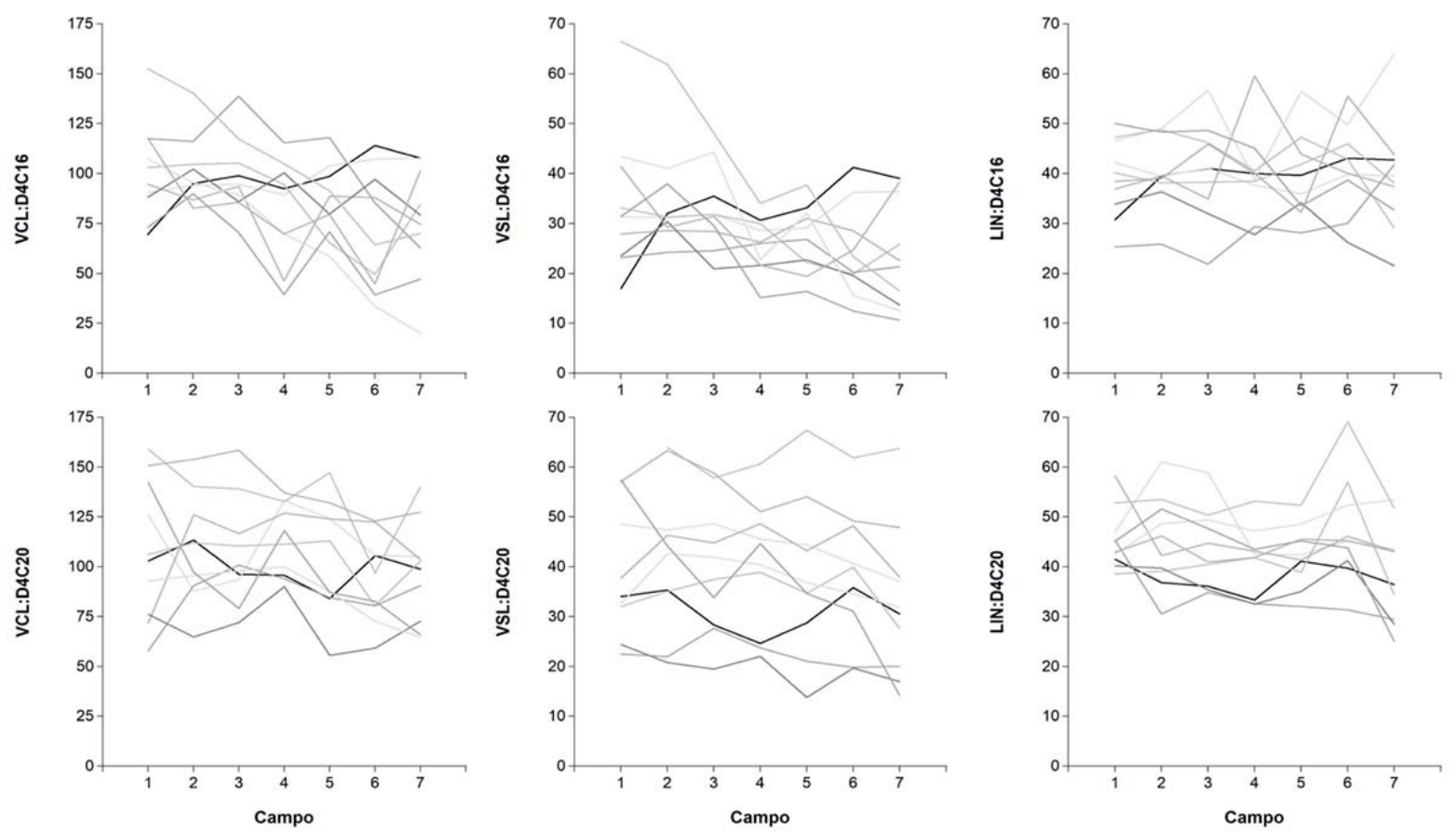

Figura 1. Efecto de la zona de recuento sobre los valores de velocidad curvilínea (VCL, $\left.\mu \mathrm{m} . \mathrm{s}^{-1}\right)$, velocidad rectilínea $\left(\right.$ VSL, $\left.\mu \mathrm{m} . \mathrm{s}^{-1}\right)$ y linealidad (LIN, \%) en cámaras ISAS®D4C (16, $20 \mu \mathrm{m})$ en espermatozoides de diez verracos pietrain, Valencia, España. 2017. Cada linea representa los valores correspondientes a cada verraco.

Figure 1. Counting zone effect on the values of curvilinear velocity $\left(\mathrm{VCL}, \mu \mathrm{m} . \mathrm{s}^{-1}\right.$ ), rectilinear velocity (VSL, $\left.\mu \mathrm{m} . \mathrm{s}^{-1}\right)$ and linearity $(\mathrm{LIN}$, $\%)$ on ISAS®D4C cameras $(16,20 \mu \mathrm{m})$ on ten pietrain boar spermatozoa, Valencia, Spain. 2017.

Each line represents the values corresponding to each boar.

El efecto de la altura de la cámara de recuento fue significativo para todas las variables de cinética espermática. Los valores obtenidos con las cámaras D4C20 fueron superiores $(\mathrm{p}<0,05)$ que en las D4C16 (Cuadro 2).

Se observaron diferencias significativas $(\mathrm{p}<0,05)$ entre los parámetros cinéticos de los diferentes verracos. El animal 8 presentó la mayor velocidad curvilínea $\left(118,15 \pm 1,62 \mu \mathrm{m} . \mathrm{s}^{-1}\right)$, mientras que el animal 10 mostró el valor menor de velocidad $\left(68,16 \pm 3,38 \mu \mathrm{m} . \mathrm{s}^{-1}\right)$, ambos medidos a una altura de cámara de $16 \mu \mathrm{m}$. En las estimaciones de VCL a $20 \mu \mathrm{m}$, el animal 8 también mostró el mayor valor $\left(140,42 \pm 1,53 \mu \mathrm{m} \cdot \mathrm{s}^{-1}\right)$, mientras que el animal 6 el menor $\left(70,78 \pm 1,85 \mu \mathrm{m} \cdot \mathrm{s}^{-1}\right)$. Cuando se compararon las alturas de cámara por animal tan solo los animales 5 y 6 presentaron mayores velocidades curvilíneas con la cámara D4C16, respecto de la D4C20 (Cuadro 3). 
Cuadro 2. Parámetros de cinética espermática (medias $\pm E E$ ) en espermatozoides de verracos pietrain, medidos con diferentes alturas de cámara de recuento. Valencia, España. 2017.

Table 2. Sperm kinetics parameters (means \pm SE) on pietrain boar spermatozoa measured with different counting chamber heights. Valencia, Spain. 2017.

\begin{tabular}{ccc}
\hline Parámetro/cámara & D4C16 & D4C20 \\
\hline Número de células & 11396 & 1681 \\
VCL $(\mu \mathrm{m} / \mathrm{s})$ & $95,96 \pm 0,56^{\mathrm{a}}$ & $38,96 \pm 0,43 \pm 0,15^{\mathrm{b}}$ \\
VSL $(\mu \mathrm{m} / \mathrm{s})$ & $31,60 \pm 0,18^{\mathrm{a}}$ & $56,34 \pm 0,24^{\mathrm{b}}$ \\
VAP $(\mu \mathrm{m} / \mathrm{s})$ & $50,06 \pm 0,29^{\mathrm{a}}$ & $42,68 \pm 0,19^{\mathrm{b}}$ \\
LIN $(\%)$ & $39,49 \pm 0,23^{\mathrm{a}}$ & $71,34 \pm 0,18^{\mathrm{b}}$ \\
STR $(\%)$ & $67,44 \pm 0,22^{\mathrm{a}}$ & $56,89 \pm 0,15^{\mathrm{b}}$ \\
WOB $(\%)$ & $55,86 \pm 0,18^{\mathrm{a}}$ & $1,45 \pm 0,004^{\mathrm{b}}$ \\
ALH $(\mu \mathrm{m})$ & $1,37 \pm 0,005^{\mathrm{a}}$ & $20,12 \pm 0,10^{\mathrm{b}}$ \\
BCF $(\mathrm{Hz})$ & $16,80 \pm 0,11^{\mathrm{a}}$ & \\
\hline
\end{tabular}

VCL: velocidad curvilínea; VSL: velocidad rectilínea; VAP: velocidad media; LIN: índice de linealidad; STR: índice de rectitud; WOB: índice de oscilación; ALH: desplazamiento lateral de la cabeza; BCF: frecuencia de entrecruzamiento. ${ }^{\text {ab }}$ Dentro de fila, valores con diferente superíndice presentan diferencias significativas $(\mathrm{p}<0,05)$ / VCL: curvilinear velocity; VSL: rectilinear velocity; VAP: average velocity; LIN: linearity index; STR: straightness index; WOB: oscillation index; ALH: lateral head displacement; BCF: cross-linking frequency. ${ }^{a, b}$ Within row, values with different superscripts present significant differences $(\mathrm{p}<0.05)$.

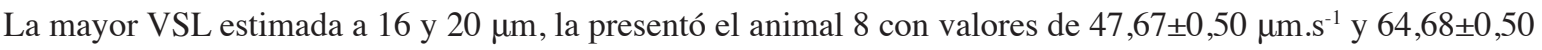
$\mu \mathrm{m} . \mathrm{s}^{-1}$, respectivamente, mientras que la menor velocidad rectilínea, y para ambas alturas de cámara, la presentó

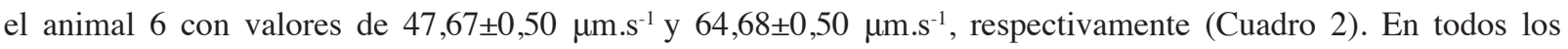
animales, excepto el 1, 5 y 6, hubo una mayor VSL en las cámaras con una altura de $20 \mu \mathrm{m}$. La VAP mostró un patrón similar que la VCL y la VSL. Para los índices LIN, STR y WOB, el animal 8 presentó los mayores valores, mientras que los valores menores para LIN y STR los obtuvo el animal 5, y el animal 6 presentó los valores más bajos de oscilación. Cuando se compararon las diferencias entre las alturas de cámara para los índices, la cámara D4C20 mostró los valores mayores en todos los animales excepto el animal 1 para los índices LIN y STR, mientras que en el animal 5 la STR estimada a $16 \mu \mathrm{m}$ fue mayor con respecto a la altura de $20 \mu \mathrm{m}$. Los valores estimados de ALH con las cámaras de 16 y $20 \mu \mathrm{m}$, fueron mayores en los animales 5 y 9, con valores de 1,66 $\pm 0,01 \mu \mathrm{m}$ y $1,67 \pm 0,01 \mu \mathrm{m}$, respectivamente, mientras que los valores más bajos fueron mostrados por los animales 10 y 6 , con valores de $1,13 \pm 0,03 \mu \mathrm{m}$ y $1,13 \pm 0,02 \mu \mathrm{m}$, respectivamente. Esta tendencia de valores bajos la presentaron los mismos animales para la BCF. Los valores mayores de BCF para ambas alturas de cámara, los mostró el animal 8 con valores de $22,91 \pm 0,31 \mathrm{~Hz}$ y $30,76 \pm 0,33 \mathrm{~Hz}$, respectivamente. Cuando se compararon las diferentes alturas de cámara por animal, tanto para la $\mathrm{ALH}$ como para la $\mathrm{BCF}$, los valores fueron mayores con la cámara D4C20, excepto para los animales 5 y 6 que presentaron mayores valores con la cámara de $16 \mu \mathrm{m}$ de altura (Cuadro 2). 


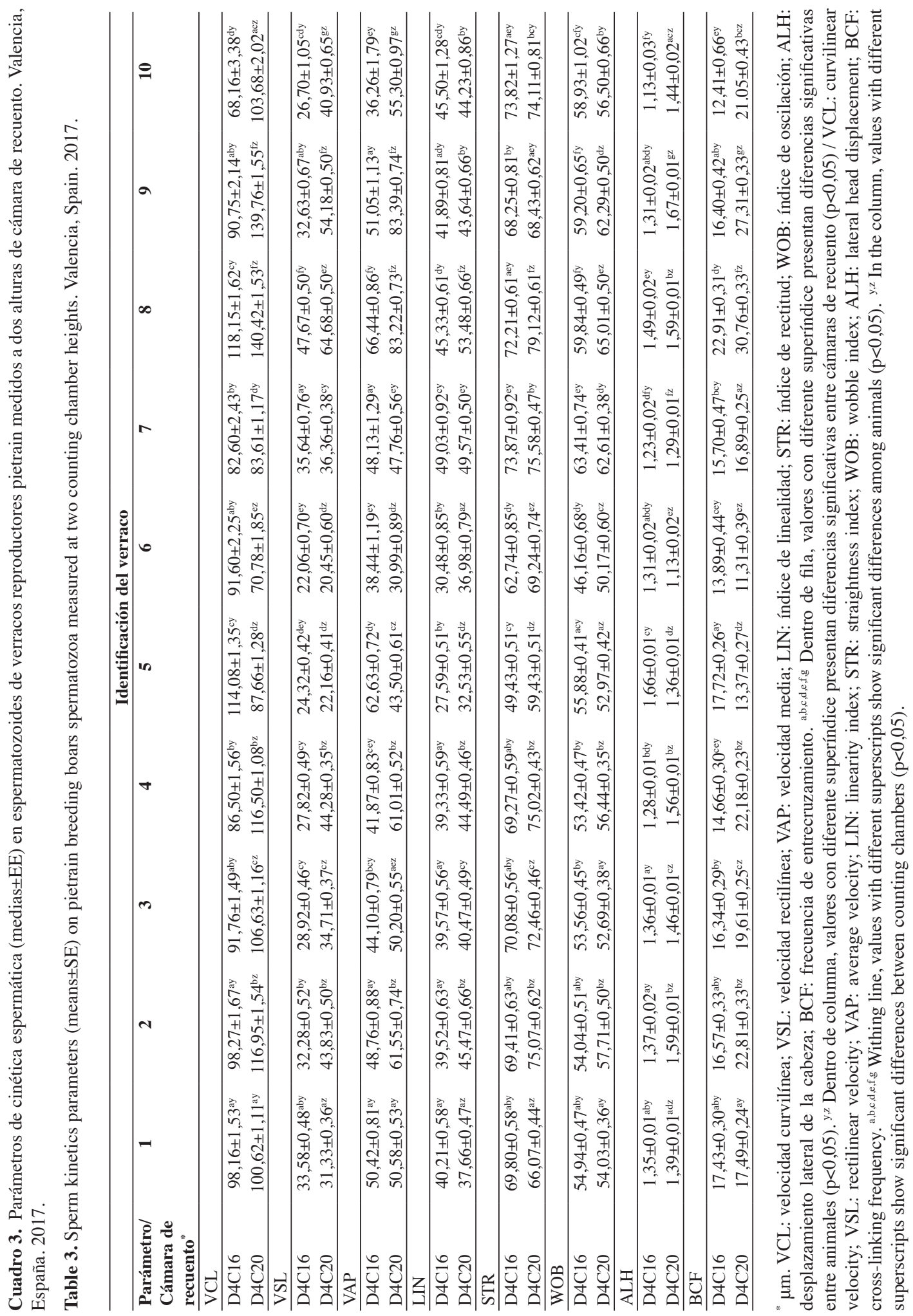




\section{Discusión}

Aunque el efecto de la zona de análisis dentro de la cámara de recuento fue significativo no, hubo diferencias en la prueba de comparación de medias para la velocidad curvilínea y rectilínea, entre los primeros tres campos de análisis para la cámara de $16 \mu \mathrm{m}$ de altura. Con respecto a la cámara de $20 \mu \mathrm{m}$, los primeros cuatro campos de análisis no mostraron diferencias significativas. Las diferencias significativas presentadas entre los primeros campos de análisis respecto de los últimos (campos seis y siete) se podrían atribuir a la presencia de movimiento pasivo (drifting) y también a un efecto aleatorio inducido por el individuo, lo que sugiere que el diseño de las cámaras es uniforme y los resultados no se alteran per se. Las estandarizaciones de la Organización Mundial de la Salud (OMS), aunque se refieren a espermatozoides humanos, recomiendan el uso de cámaras de $20 \mu \mathrm{m}$ (World Health Organization. 2010), debido a que el desplazamiento de los espermatozoides se produce como consecuencia del movimiento flagelar con una amplitud aproximada de $17 \mu \mathrm{m}$ (David et al., 1981). En especies domésticas no ha habido un estudio sistemático que proponga o recomiende condiciones técnicas para estandarizar el análisis seminal con sistemas CASA.

La mayoría de las cámaras desechables disponibles en el mercado se basan en el principio de carga por acción capilar, y comúnmente incluyen una tapa deslizante que se fija con diferentes tipos de pegamento. En la fase de fabricación, el pegamento se usa no solo para diseñar la forma de la cámara de recuento, sino también para definir su profundidad, dependiendo de la altura del pegamento (Bompart et al., 2018). Los diferentes diseños de cámara afectan tanto la distribución final de los espermatozoides dentro de la cámara como las características de movilidad y cinética. Esto se ha demostrado en diversas especies como el verraco (Christensen et al., 2005), el bovino (Prathalingam et al., 2006; Contri et al., 2010; Lenz et al., 2011; Gloria et al., 2013), el caprino (Del-Gallego et al., 2017), el humano (Tomlinson et al., 2001; Peng et al., 2015), conejo (Massányi et al., 2008), ovino (Palacín et al., 2013) y el equino (Len et al., 2010; Spizziri et al., 2010; Hoogewijs et al., 2012). En especies como la caprina, la movilidad progresiva y las velocidades de los espermatozoides fueron mayores con cámaras de carga capilar a alturas de $20 \mu \mathrm{m}$ respecto de $10 \mu \mathrm{m}$ (Del-Gallego et al., 2017). Sin embargo, estos resultados no se confirmaron en otras especies, como la humana (Lannou et al., 1992; Soler et al., 2012) o el hámster (Cricetinae), (Shivaji et al., 1995), lo que podría relacionarse con el hecho de que los espermatozoides de diferentes especies exhiben diferentes patrones de movilidad.

Los resultados obtenidos en el presente trabajo mostraron diferencias significativas entre las diferentes alturas evaluadas en las cámaras desechables, y se incrementó el valor de cada parámetro cinemático estimado a una altura de cámara mayor. Otros trabajos realizados en semen humano, mostraron diferencias en concentración espermática y movilidad total cuando se utilizaron cámaras de recuento reutilizables a alturas de 10 y $20 \mu \mathrm{m}$ (Makler, 1978). Por el contrario, en semen humano (Soler et al., 2012) no se observaron diferencias en parámetros espermáticos, como la concentración y la movilidad total, cuando se utilizaron cámaras ISASD4C (alturas de 10, 16 y $20 \mu \mathrm{m}$ ), lo que sugiere que el movimiento espermático es característico de cada especie $\mathrm{y}$, además, que se debe considerar el método de carga de las cámaras de recuento. Esto sugiere que al haber más amplitud dentro de la cámara de recuento y por consiguiente, mayor capacidad de volumen, las células podrían desplazarse y realizar su movimiento sin restricciones, lo que explicaría el incremento en los valores cinéticos conforme se incrementa la altura de la cámara de recuento.

Otro factor que podría explicar los resultados obtenidos en el presente trabajo, es que las cámaras de recuento se cargan por el progreso capilar entre las dos capas (deslizamiento y cubierta), bajo una fuerza derivada de la tensión superficial entre el fluido y el vidrio, formando un menisco en el borde delantero o flujo laminar de Poiseuille (efecto Segre-Silberberg, SS) (Douglas-Hamilton et al., 2005). Este efecto depende de la altura de las cámaras, porque la velocidad de llenado es proporcional a la altura y disminuye a medida que el menisco penetra en la cámara, por lo tanto, las cámaras poco profundas se llenan más lentamente que las más profundas, lo que proporciona una posible explicación de las diferencias entre cámaras de diferentes alturas (Kuster, 2005) en el 
sentido de que, a mayor altura de la cámara, mejor es la distribución uniforme. Además, el llenado de una cámara por acción capilar expone las partículas suspendidas en el fluido a un gradiente de velocidad, lo que hace que las partículas migren perpendicularmente a la dirección del flujo (Vasseur y Cox, 1976).

En función de la especie y el estado fisiológico, se necesita un espacio considerable para desarrollar correctamente la movilidad espermática, debido a que el movimiento natural de los espermatozoides es helicoidal (Kraemer et al., 1998; Soler et al., 2018). En la literatura veterinaria, la altura de la cámara de recuento para el análisis de la cinética y movilidad del semen es variable y por lo general, no se suele mencionar (Hoogewijs et al., 2012). El uso de cámaras con mayor profundidad hace que el efecto SS sea insignificante y en una cámara de 20 $\mu \mathrm{m}$ los espermatozoides de verraco alcanzarían sus planos estables del efecto SS con mayor rapidez después de que el fluido se haya movido $1 \mathrm{~mm}$ hacia la cámara de recuento (Kuster, 2005).

\section{Conclusiones}

Los resultados obtenidos a diferentes alturas de cámara de recuento (16 y $20 \mu \mathrm{m}$ ) fueron precisos y repetibles en el análisis seminal. No obstante, los resultados obtenidos en parámetros de cinética espermática a $20 \mu \mathrm{m}$ fueron superiores respecto de $16 \mu \mathrm{m}$, lo que sugiere que las células se pueden mover sin restricción en una cámara de recuento a una altura mayor. En el efecto de zona, el primer campo de captura (zona 1) tendió a presentar mayores velocidades que los subsiguientes campos como consecuencia del movimiento pasivo o drifting. Este trabajo en verracos aporta nueva información con respecto a la utilización de cámaras de recuento en el análisis seminal y debe continuarse con otros estudios que incluyan comparaciones entre diferentes sistemas CASA, con el propósito de estandarizar métodos y permitir relacionar adecuadamente los resultados.

\section{Literatura citada}

Amann, R., and D. Waberski. 2014. Computer-assisted sperm analysis (CASA): Capabilities and potential developments. Theriogenology 81(1):5-17. doi:10.1016/j.theriogenology.2013.09.004

Bompart, D., A. García-Molina, A. Valverde, C. Caldeira, J. Yániz, M. Núñez-de-Murga, and C. Soler. 2018. CASA-Mot technology: how results are affected by the frame rate and counting chamber. Reprod. Fertil. Dev. 30:810-819. doi:10.1071/RD17551

Broekhuijse, M.L., E. Šoštarić, H. Feitsma, and B.M. Gadella. 2011. Additional value of computer assisted semen analysis (CASA) compared to conventional motility assessments in pig artificial insemination. Theriogenology 76:1473-1486. doi:10.1016/j.theriogenology.2011.05.040

Christensen, P., H. Stryhn, and C. Hansen. 2005. Discrepancies in the determination of sperm concentration using Bürker-Türk, Thoma and Makler counting chambers. Theriogenology 63:992-1003. doi:10.1016/J.THERIOGENOLOGY.2004.05.026

Contri, A., C. Valorz, M. Faustini, L. Wegher, and A. Carluccio. 2010. Effect of semen preparation on casa motility results in cryopreserved bull spermatozoa. Theriogenology 74:424-435. doi:10.1016/j.theriogenology.2010.02.025.

David, G., C. Serres, and P. Jouannet. 1981. Kinematics of human spermatozoa. Mol. Reprod. Dev. 4(2):83-95. doi:10.1002/ mrd.1120040202

Del-Gallego, R., S. Sadeghi, E. Blasco, C. Soler, J.L. Yániz, and M.A. Silvestre. 2017. Effect of chamber characteristics, loading and analysis time on motility and kinetic variables analysed with the CASA-mot system in goat sperm. Anim. Reprod. Sci. 177:97-104. doi:10.1016/j.anireprosci.2016.12.010 
Douglas-Hamilton, D.H., N.G. Smith, C.E. Kuster, J.P. Vermeiden, and G.C. Althouse. 2005. Capillary-loaded particle fluid dynamics: effect on estimation of sperm concentration. J. Androl. 26(1):115-22.

Ehlers, J., M. Behr, H. Bollwein, M. Beyerbach, and D. Waberski. 2011. Standardization of computer-assisted semen analysis using an e-learning application. Theriogenology 76:448-454. doi:10.1016/j.theriogenology.2011.02.021

Gill, H.S., K. Arsdalen, J. Hypolite, R.M. Levin, and J.V. Ruzich. 1988. Comparative study of two computerized semen motility analyzers1: vergleichende studie an zwei verschiedenen computer-samen-analysern. Andrologia 20:433-440. doi:10.1111/j.1439-0272.1988.tb00721.x

Gloria, A., A. Carluccio, A. Contri, L. Wegher, C. Valorz, and D. Robbe. 2013. The effect of the chamber on kinetic results in cryopreserved bull spermatozoa. Andrology 1:879-885. doi:10.1111/j.2047-2927.2013.00121.x

Hoogewijs, M.K., S.P. de-Vliegher, J.L. Govaere, C. De Schauwer, A. De Kruif, and A. Van-Soom. 2012. Influence of counting chamber type on CASA outcomes of equine semen analysis. Equine. Vet. J. 44:542-549 doi:10.1111/j.20423306.2011.00523.x

Jasko, D.J., D.H. Lein, and R.H. Foote. 1990. A comparison of two computer-assisted semen analysis instruments for the evaluation of sperm motion characteristics in the stallion. J. Androl. 11:453-459. doi:10.1002/j.1939-4640.1990.tb00176.X

Kraemer, M., C. Fillion, B. Martin-Pont, and J. Auger. 1998. Factors influencing human sperm kinematic measurements by the celltrak computer-assisted sperm analysis system. Hum. Reprod. 13(3):611-619. doi:10.1093/humrep/13.3.611

Krause, W. 1995. Computer-assisted semen analysis systems: comparison with routine evaluation and prognostic value in male fertility and assisted reproduction. Hum. Reprod. 1:60-66. doi:10.1093/humrep/10.suppl_1.60

Kuster, C. 2005. Sperm concentration determination between hemacytometric and CASA systems: Why they can be different? Theriogenology 64:614-617. doi:10.1016/J.THERIOGENOLOGY.2005.05.047

Lannou, D., J.F. Griveau, J.P. Pichon, and J.C. Le-Quero. 1992. Effects of chamber depth on the motion pattern of human spermatozoa in semen or in capacitating medium. Hum. Reprod. 7:1417-1421. doi:10.1093/oxfordjournals.humrep. a137585

Len, J.A., J.A. Jenkins, B.E. Eilts, D.L. Paccamonti, S.K. Lyle, and G. Hosgood. 2010. Immediate and delayed (after cooling) effects of centrifugation on equine sperm. Theriogenology 73:225-231. doi:10.1016/J.THERIOGENOLOGY.2009.09.003

Lenz, R.W., M.E. Kjelland, K. VonderHaar, T.M. Swannack, and J.F. Moreno. 2011. A comparison of bovine seminal quality assessments using different viewing chambers with a computer-assisted semen analyzer. J. Anim. Sci. 89:383-388. doi: $10.2527 /$ jas.2010-3056

Loomis, P.R., and J.K. Graham. 2008. Commercial semen freezing: Individual male variation in cryosurvival and the response of stallion sperm to customized freezing protocols. Anim. Reprod. Sci. 105:119-128. doi:10.1016/J. ANIREPROSCI.2007.11.010

Makler, A. 1978. A new chamber for rapid sperm count and motility estimation. Fertil. Steril. 30:313-318. doi:10.1016/S00150282(16)43518-1

Massányi, P., P. Chrenek, N. Lukáč, A. Makarevich, A. Ostro, P. Šafarik, J. Živčak, and J. Bulla. 2008. Comparison of different evaluation chambers for analysis of rabbit spermatozoa motility parameters using CASA system. Slovak. J. Anim. Sci. 41(2):60-66.

Olds-Clarke, P., H.M. Baer, and W.L. Gerber. 1990. Human sperm motion analysis by automatic (Hamilton-Thorn Motility Analyzer) and manual (Image-80) digitization systems. J. Androl. 11:52-58. 
Ortega-Ferrusola, C., B. Macías, V. Suárez, J. Gallardo-Bolaños, L. González-Fernández, J. Tapia, H. Rodríguez, and F. Peña. 2009. Identification of sperm subpopulations in stallion ejaculates: changes after cryopreservation and comparison with traditional statistics. Reprod. Domest. Anim. 44:419-423. doi:10.1111/j.1439-0531.2008.01097.x

Palacín, I., S. Vicente-Fiel, P. Santolaria, and J.L. Yániz. 2013. Standardization of CASA sperm motility assessment in the ram. Small Ruminant Res. 112:128-135. doi:10.1016/j.smallrumres.2012.12.014

Peng, N., X. Zou, and L. Li. 2015. Comparison of different counting chambers using a computer-assisted semen analyzer. Syst. Biol. Reprod. Med. 61:307-313. doi:10.3109/19396368.2015.1063175

Prathalingam, N.S., W.V. Holt, S.G. Revell, S. Jones, and P.F. Watson. 2006. The precision and accuracy of six different methods to determine sperm concentration. J. Androl. 27:257-262. doi: 10.2164/jandrol.05112

Rijsselaere, T., A. Van-Soom, D. Maes, and A. Kruif. 2003. Effect of technical settings on canine semen motility parameters measured by the Hamilton-Thorne analyzer. Theriogenology 60:1553-1568. doi:10.1016/S0093-691X(03)00171-7

Shivaji, S., J. Peedicayil, and L.G. Devi. 1995. Analysis of the motility parameters of in vitro hyperactivated hamster spermatozoa. Mol. Reprod. Dev. 42:233-247. doi:10.1002/mrd.1080420213

Simonik, O., J. Sichtar, A. Krejcarkova, R. Rajmon, L. Stadnik, J. Beran, M. Dolezalova, and Z. Biniova. 2015. Computer assisted sperm analysis - the relationship to bull field fertility, possible errors and their impact on outputs: a review. Indian J. Anim. Sci. 85:3-11.

Smith, S.C., and G.C. England. 2001. Effect of technical settings and semen handling upon motility characteristics of dog spermatozoa measured using computer-aided sperm analysis. J. Reprod. Fertil. Suppl. 57:151-9.

Soler, C., M. Fuentes, M. Sancho, A. García, M. Núñez-de-Murga, and J. Núñez-de-Murga. 2012. Effect of counting chamber on seminal parameters, analyzing with the ISASv1@. Rev. Int. Androl. 10(4):132-138. doi:10.1016/S1698-031X(12)70069-9

Soler, C., J. Picazo-Bueno, V. Micó, A. Valverde, D. Bompart, F.J. Blasco, J.G. Álvarez, and A. García-Molina. 2018. Effect of counting chamber depth on the accuracy of lensless microscopy for the assessment of boar sperm motility. Reprod. Fertil. Dev. 30:924-934. doi:10.1071/RD17467

Spizziri, B.E., M.H. Fox, J.E. Bruemmer, E.L. Squires, and J.K. Graham. 2010. Cholesterol-loaded-cyclodextrins and fertility potential of stallions spermatozoa. Anim. Reprod. Sci. 118:255-264. doi:10.1016/J.ANIREPROSCI.2009.08.001

Tejerina, F., K. Buranaamnuay, F. Saravia, M. Wallgren, and H. Rodriguez-Martinez. 2008. Assessment of motility of ejaculated, liquid-stored boar spermatozoa using computerized instruments. Theriogenology 69:1129-1138. doi:10.1016/j. theriogenology.2008.01.027

Tomlinson, M., J. Turner, G. Powell, and D. Sakkas. 2001. One-step disposable chambers for sperm concentration and motility assessment: how do they compare with the World Health Organization's recommended methods? Hum. Reprod. 16(1):121-124. doi:10.1093/humrep/16.1.121

Vasseur, P., and R.G. Cox. 1976. The lateral migration of a spherical particle in two-dimensional shear flows. J. Fluid Mechanics 78:385-413. doi:10.1017/S0022112076002498

Verstegen, J., M. Iguer-Ouada, and K. Onclin. 2002. Computer assisted semen analyzers in andrology research and veterinary practice. Theriogenology 57:149-79. doi:10.1016/S0093-691X(01)00664-1

Waite, J.A., C.C. Love, S.P. Brinsko, S.R. Teague, J.L. Salazar, S.S. Mancill, and D.D. Varner. 2008. Factors impacting equine sperm recovery rate and quality following cushioned centrifugation. Theriogenology 70:704-714. doi:10.1016/j. theriogenology.2008.04.047

World Health Organization. 2010. WHO laboratory manual for the examination and processing of human semen. $5^{\text {th }}$ ed. World Health Organization, Geneva, SUI. 